\title{
Crystalline Covalent Organic Framework Aerogels
}

\author{
Dongyang Zhu'\#, Yifan Zhu2\#, Qianqian Yan², Fangxin Liu, Pingfeng Yu³, Chia-Ping Tseng1, Nicho- \\ las Tjahjono ${ }^{2}$, Po-Chun Huang1, Muhammad M. Rahman², Eilaf Egap², Pulickel M. Ajayan², and Ra- \\ fael Verduzco ${ }^{1,2 *}$
}

\author{
${ }^{1}$ Department of Chemical and Biomolecular Engineering, Rice University, Houston, Texas 77005, USA \\ ${ }^{2}$ Department of Materials Science and NanoEngineering, Rice University, Houston, Texas 77005, USA \\ ${ }^{3}$ Departmentof Civil and Environmental Engineering, Rice University, Houston, Texas 77005, USA \\ \#D. Zhu and Y. Zhu contributed equally
}

\begin{abstract}
Covalent organic frameworks (COFs) are crystalline organic materials of interest for a wide range of applications due to their porosity, tunable architecture, and precise chemistry. However, COFs are typically produced in powder form and are difficult to process. Herein, we report a simple and versatile approach to fabricate macroscopic, crystalline COF gels and aerogels. Our method involved the use of dimethyl sulfoxide as a solvent and acetic acid as a catalyst to first produce a COF gel. The COF gel was then washed, dried, and reactivated to produce a macroscopic, crystalline, porous COF aerogel. We demonstrated this synthesis for six different imine COFs and found that the crystallinities and porosities of the COF aerogels matched those of COF powders. Electron microscopy revealed a robust hierarchical pore structure, and we showed that the COF aerogels can be used as absorbents in oil-water separations, for the removal of organic and inorganic micropollutants, and for the capture and retention of iodine. This study provides a versatile and simple approach for the fabrication of $\mathrm{COF}$ aerogels and will provide novel routes for incorporating COFs in applications that require macroscopic, porous materials.
\end{abstract}

\section{- INTRODUCTION}

Covalent organic frameworks (COFs) are an emerging type of porous material synthesized by dynamic, covalent bonds. ${ }^{1-3}$ Due to their tunable chemistry, tailorable structures, regular pore channels and high surface areas, COFs have received significant interest for applications including membrane separation, pollutant removal, and energy storage $^{1,3}$. However, most COFs are produced in the form of powders that are difficult to process. ${ }^{4}$ This limits the testing and development of COFs for many applications including membrane-based separations, electrochemical processes that rely on separators or electrodes, and adsorption processes that require easy removal or separation of the adsorbent. ${ }^{2,4-11}$

Recent studies have demonstrated novel processing and synthesis methods for producing macroscopic COF films and foams. Dichtel and coworkers reported an acidmediated exfoliation method that enables the dissolution of COF powders and solvent processing of COFs into thin films. ${ }^{4}$ In another example, Banerjee and coworkers developed an organic terracotta process and were able to process COF powders into sculptures using $p$-toluenesulfonic acid (PTSA) as the organizer. ${ }^{11}$ However, the synthesis and processing of macorscopic COFs for applications remains a significant challenge.

Aerogels or foams are of particular interest for applications in adsorption and separation fields because they pro- vide high surface areas and an interconnected network of pores. The presence of different types of pore sizes enhances molecular accessibility due to interconnected pore channels and facilitates a high rate of mass transport.6,12 Pioneering work by Banerjee and coworkers described the fabrication of a series of $\beta$-ketoenamine COF foams through an in-situ gas-phase foaming protocol. The asfabricated COF foams showed hierarchical porosity and superior dye adsorption efficiency compared with COF powders. ${ }^{6}$ Thomas and coworkers successfully fabricated $\mathrm{COF} / g r a p h e n e$ aerogels through a hydrothermal approach, where COF monomers and reduced graphene oxide (rGO) were reacted at elevated temperatures to form wet gels and then freeze-dried to produce ultralight aerogel. ${ }^{12}$ The composite aerogel exhibited a high capacity for adsorption of organic solvents adsorption and superb electrochemical supercapacitor performance. Despite these recent advances, we still lack straightforward and versatile methods to fabricate crystalline and porous COF aerogels.

Herein, we demonstrate a simple approach to synthesize pure crystalline COF aerogels using dimethyl sulfoxide (DMSO) as the reaction solvent and 6M acetic acid as the catalyst (see Figure 1). This solvent and catalyst combination produced a uniform wet gel, and COF synthesis was conducted in the gelled state. After reaction, the gel was washed, dried, and activated to produce crystalline and porous COF aerogels. These macroscopic COF aerogels retain the shape of the reaction vial, feature hierarchical 


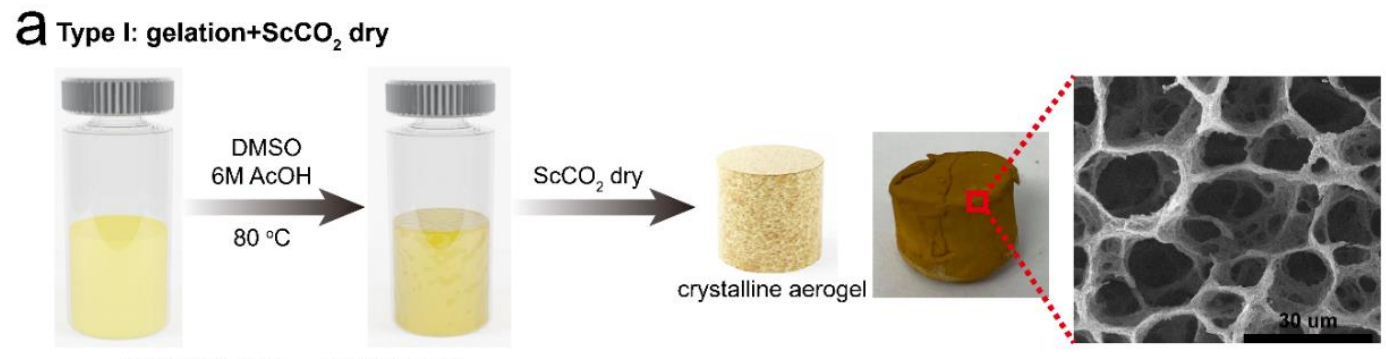

TAPB-OMePDA and TAPB-TFPA

Type II:gelation $+\mathrm{ScCO}_{2}$ dry+reactivation $+\mathrm{ScCO}_{2}$ dry

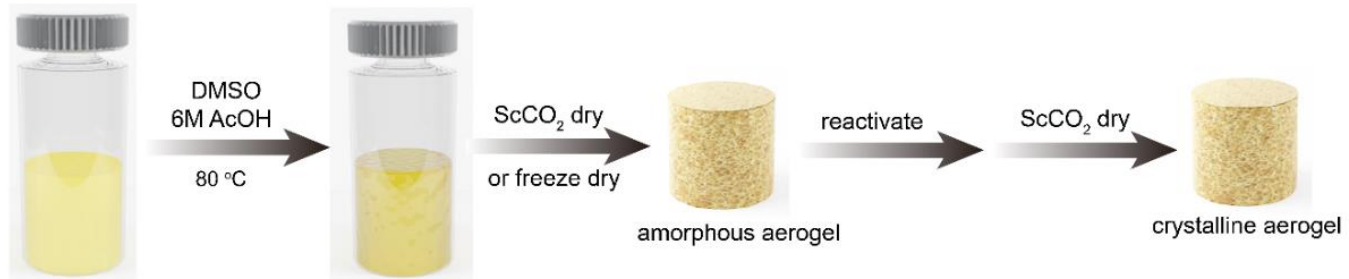

TAPA-TFPA,TAPB-PDA, TAPB-BrPDA and BPDA-BTCA

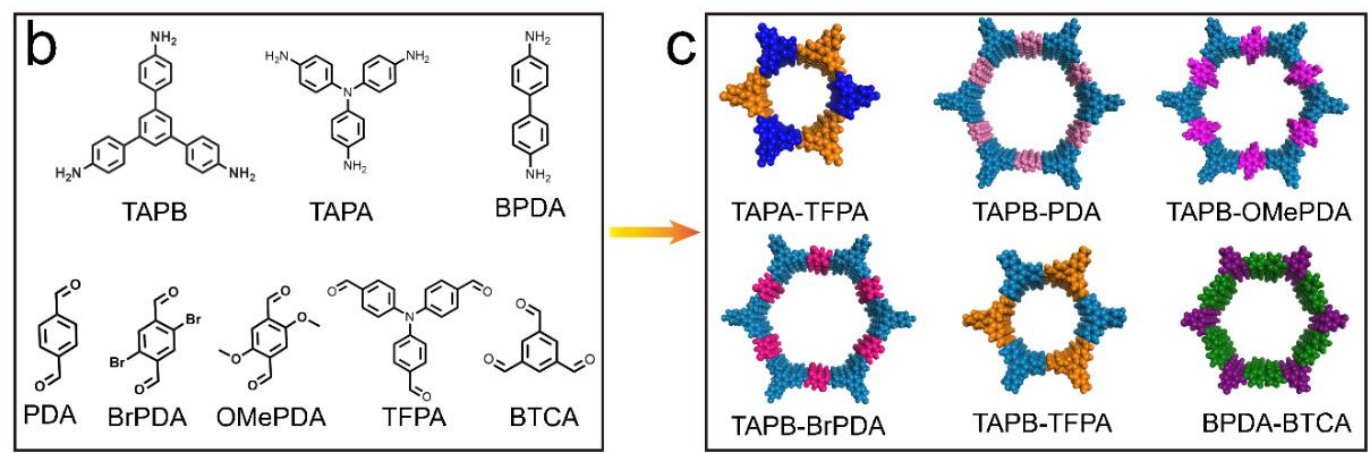

Figure 1. (a) Schematics for fabrication of COF aerogel through gelation and supercritical $\mathrm{CO}_{2}$ drying (Type I) or through gelation, reactivation, and supercritical $\mathrm{CO}_{2}$ drying (Type II). (b) COF monomers used in this study, and (c) Schematics for six different COF chemistries studied. (TAPB: tris(4-aminophenyl)benzene; TAPA: tris(4-aminophenyl)amine; BPDA: (1,1'-Biphenyl])-4,4'diamine; PDA: terephthaldehyde; BrPDA: 2,5-dibromoterephthalaldehyde; OMePDA: 2,5-dimethoxyterephthalaldehyde; TFPA: tris(4-formylphenyl)amine; BTCA: 1,3,5-benzenetricarboxaldehyde).

micropores and mesopores, and show excellent adsorption capacities. We demonstrated that these COFs could be easily effectively implemented in adsorption and decontamination applications. This work is significant because it provides a straightforward and general route to produce macroscopic COF aerogels, and our method will be of interest to those interested in developing COFs for membranebased separations, energy storage applications, decontamination and remediation, and other applications which benefit from macroscopic and porous COF scaffolds.

\section{RESULTS AND DISCUSSION}

Synthesis and characterizations of COF aerogels. To test our procedure for preparing COF aerogels, we targeted six different COF chemistries using the monomers shown in Figure $1 \mathrm{~b}$. In the first step of the synthesis, a stoichiometric ratio of amino monomers (TAPB, TAPA, or BPDA) and aldehyde monomers (PDA, BrPDA, OMePDA, or TFPA) were dissolved in $1 \mathrm{~mL}$ DMSO by sonication until a clear solution formed. Next, we added $6 \mathrm{M} \mathrm{AcOH}$ as a catalyst, and the solution gelled within one minute of adding the acid catalyst. The wet gels were then incubated at $80^{\circ} \mathrm{C}$ for 12 hours to complete the reaction, during which the solu- tion became a hazy gel. After the reaction was complete, the gels were sequentially immersed in tetrahydrofuran (THF), acetone and ethanol to remove impurities, and then they were dried under supercritical $\mathrm{CO}_{2}\left(\mathrm{ScCO}_{2}\right)$. For two chemistries studied (TAPB-OMePDA and TAPB-TFPA, Type I in Figure 1a), this process produced crystalline COF aerogels. However, TAPA-TFPA, TAPB-PDA, TAPB-BrPDA, and BPDA-BTCA COFs were amorphous aerogels after this process (Type II in Figure 1a). To produce crystalline COFs for these chemistries, these four samples were reactivated in a mixture of dioxane and mesitylene, solvent exchanged, and dried under $\mathrm{ScCO}_{2}$. The $\mathrm{COF}$ aerogels retained their macroscopic size and shape during the reactivation process. One point worth mentioning is that the first-step drying method can either be freeze drying or $\mathrm{ScCO}_{2}$ drying since amorphous samples gain crystallinity after reactivation. More details on the synthesis are provided in the Supporting Information. 
a

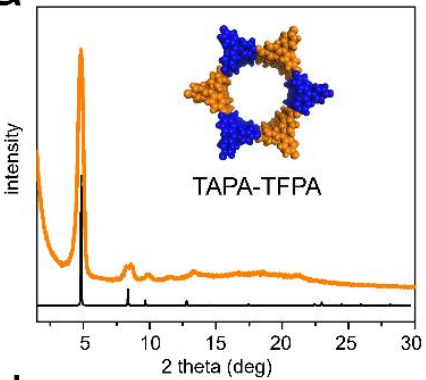

d

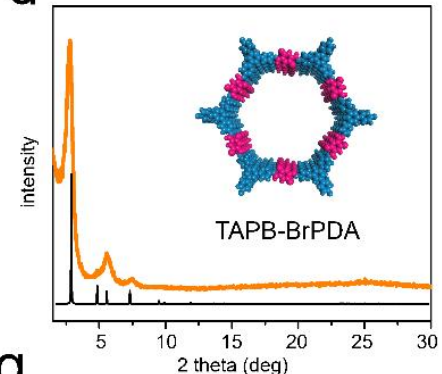

g

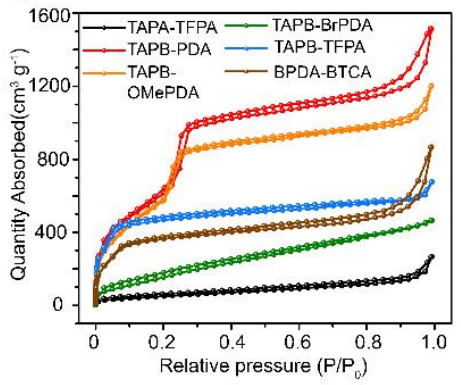

b

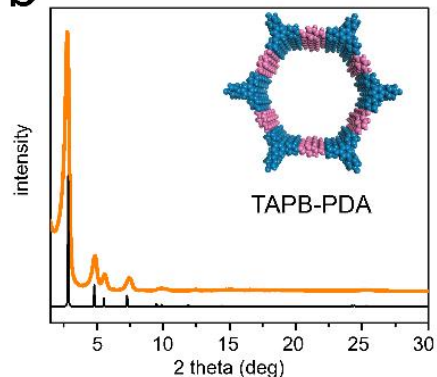

e

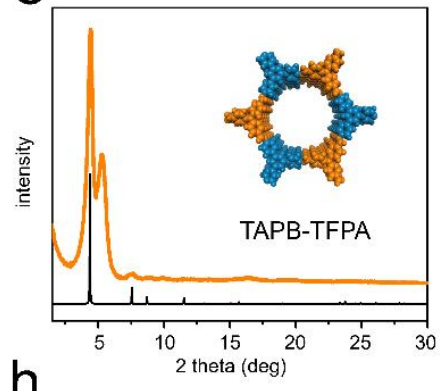

h

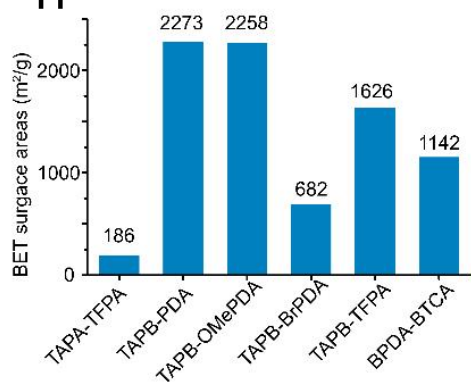

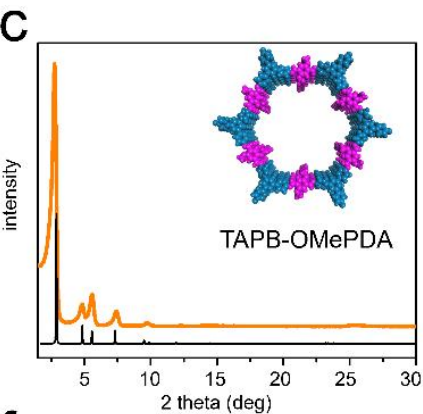

f

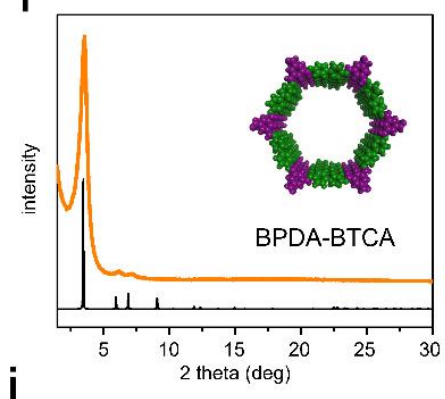

i

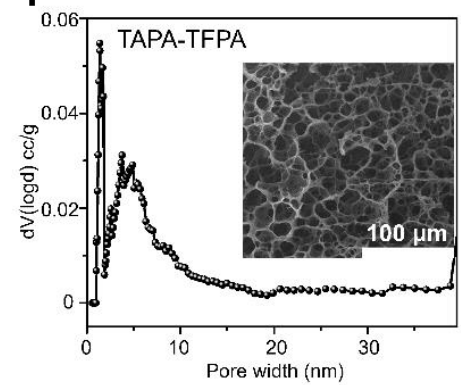

Figure 2. Characterization of COF aerogels by PXRD (a-g) and nitrogen sorption (g - i). (a-g) PXRDs for six different COFs: (a) TAPA-TFPA (b) TAPB-PDA (c)TAPB-OMePDA (d) TAPB-BrPDA (e) TAPB-TFPA (f) BPDA-BTCA. (g) Nitrogen sorption isotherms for six different COFs. (h) Calculated BET surface areas. (i) Pore size distributions for TAPA-TFPA COF aerogel. Insert shows the SEM micrograph of TAPB-TFPA COF aerogel.

The COF aerogels were characterized by powder X-ray diffraction (PXRD) analysis, Fourier-transform infrared spectroscopy (FTIR), nitrogen sorption measurements, and scanning electron microscopy (SEM) (Figure 2 and Figure S5-S14). All six COF aerogels exhibited excellent crystallinities, and the peak positions were consistent with previous reports on COF powders (Figure 2a-g). They also matched simulated XRD spectra calculated using eclipsed AA-stacking modes. The simulations did not predict the peak near 5 degrees of TAPB-TFPA COF observed in experiments, but this discrepancy between experiments and simulations has been previously observed for this particular $\mathrm{COF}^{13}$ (Figure 2f). For all six COF aerogels, FTIR analysis revealed the appearance of a $\mathrm{C}=\mathrm{N}$ stretching vibration band at $1619 \mathrm{~cm}^{-1}$ and the attenuation of the aldehyde band at $1690 \mathrm{~cm}^{-1}$ after the reaction, indicating the formation of imine bonds (Figure S7). The porous structures of COF aerogels were analyzed through nitrogen sorption tests. All six COF aerogels showed high nitrogen sorption at low relative pressures $\left(P / P_{0}<0.1\right)$, indicating a microporous structure in these COFs (Figure 2g). The BET surface areas were found to be 186, 2273, 2258, 682, 1626, $1142 \mathrm{~m}^{2} \mathrm{~g}^{-1}$ for TAPA-TFPA, TAPB-PDA, TAPB-OMePDA, TAPB-BrPDA, TAPB-TFPA and BPDA-BTCA COF aerogels, respectively, which were comparable or larger compared to their counterpart powders (Figure $2 \mathrm{~h}$ and Figure S11). The TAPA-TFPA COF aerogel and powder exhibited lower surface areas (aerogel: $186 \mathrm{~m}^{2} \mathrm{~g}^{-1}$; powder: $308 \mathrm{~m}^{2} \mathrm{~g}^{-1}$ ) compared to other COF chemistries, likely due to the lower inherent crystallinity of this particular COF. TAPB-PDA and TAPB-OMePDA COF aerogels exhibited slightly higher BET surface areas compared with their powders (see Figure 2i and Figure S11). The pore size distributions were calculated using nonlinear density functional theory (NLDFT) methods, and all exhibited a combination of microporous and mesoporous structures (Figure 2i and Figure S12). Taking TAPA-TFPA COF aerogel as an example, the pore size distributions showed sharp peaks near $1.5 \mathrm{~nm}$ and broad peaks ranging from $4 \mathrm{~nm}$ to $20 \mathrm{~nm}$, indicating the presence of a wide range of pore sizes (Figure $2 \mathrm{j}$ ). The pore size near $1.5 \mathrm{~nm}$ is consistent with previous reports ${ }^{13,14}$ and represents the intrinsic micropores of TAPATFPA. SEM analysis showed that all COF aerogels were composed of fiber-like structures (insert in Figure $2 \mathrm{j}$ and Figure S13). Of particular interest, TAPA-TFPA COF aerogels showed numerous mesopores and macropores $(\sim 20$ $100 \mu \mathrm{m}$ ), and these mesopores and 

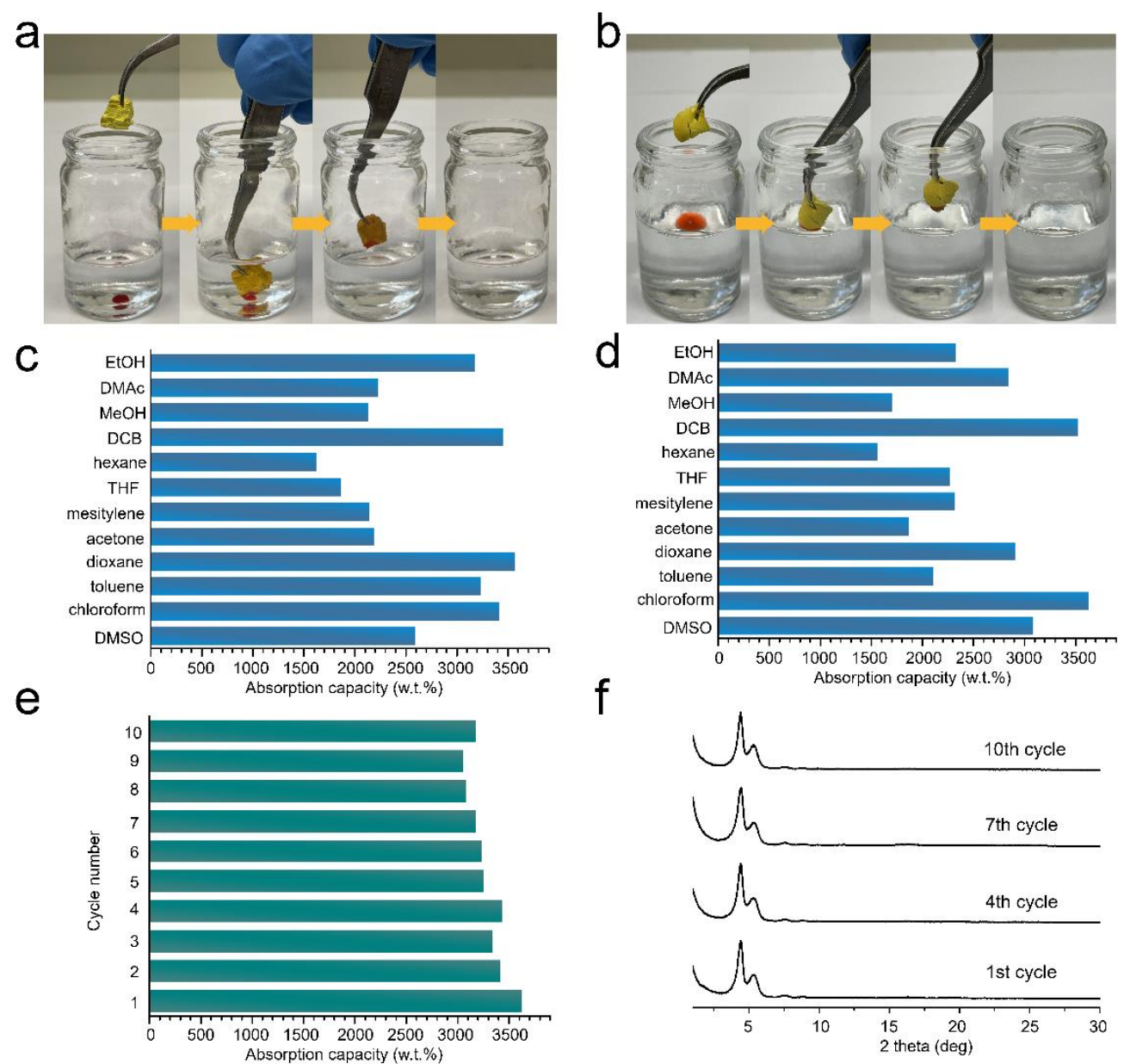

Figure 3. Summary of performance of TAPB-TFPA and TAPB-OMePDA COF aerogels for the absorption of ethanol (EtOH), dimethylacetamide (DMAc), methanol $(\mathrm{MeOH})$, dichlorobenzene (DCB), tetrahydrofuran (THF), and dimethyl sulfoxide (DMSO). (a, b) Digital photographs of absorption of (a), dyed chloroform (b), dyed silicon oil from water. (c, d) Organic solvent absorption capacities of (c) TAPB-OMePDA COF aerogel and (d) TAPB-TFPA COF aerogel. (e) Performance of TAPBTFPA COF aerogel over multiple cycles of absorption of chloroform and (f) PXRD spectra of recycled TAPB-TFPA COF after multiple cycles of absorption, washing, and drying.

macropores had a more regular structure and size when compared with the other five aerogels (Figure S13a). For the other five COF aerogels synthesized, the fiber-like structures were comprised of aggregated particles and many irregular, large voids and pores caused by the irregular stacking of the fiber-like structures (Figure S13b-f). Along with these macroscale pore structures, all COF aerogels had a crystalline, microporous structure characteristic of crystalline COFs. We also found that the amorphous aerogels exhibited the same macroporous morphology as the crystalline aerogels (Figure S14). This indicates that the 3D macroporous structure did not shrink or collapse during the reactivation process.

We further tested a variety of reaction conditions by varying the solvent, temperature, monomer concentration, catalyst amount, and drying methods (see details in Supporting Information). We found that the use of DMSO was critical to the formation of wet gels (Figure S15). We tested other solvents including dioxane, mesitylene, chloroform, THF, acetone and ethanol, but the use of these solvents produced powder samples (Figure S16). Protic solvents such as dimethylformamide, dimethylacetamide, and $\mathrm{N}$ - methyl-2-pyrrolidone were also tested in the synthesis of TAPB-PDA aerogels (see details in Supporting Information). While these solvents produced TAPB-PDA COF gels, they were not applicable to TAPB-OMePDA COFs (Figure S17). While it is unclear why DMSO produces the most uniform COF gels, hydrogen bonding between the $\mathrm{S}=0$ functional group and water, acetic acid, and the COF imine bonds may play a role in promoting the gelation process. ${ }^{15-17}$ When the reaction was conducted at ambient temperatures, a wet gel was formed, but the yield of final material was significantly lower compared to the reaction conducted at $80^{\circ} \mathrm{C}$. When the temperature was significantly higher than $80^{\circ} \mathrm{C}\left(120^{\circ} \mathrm{C}\right.$ or $\left.160^{\circ} \mathrm{C}\right)$, the wet gel shrank significantly due to the evaporation of DMSO (see details in Supporting Information). We also found that monomer concentration was an important variable. Wet gels were not observed at lower monomer concentrations (e.g. 5.5 $\mathrm{mg}$ TAPB and $3.0 \mathrm{mg}$ PDA in1 $\mathrm{mL}$ DMSO), but at higher concentrations ( $42.2 \mathrm{mg}$ of TAPB and $24.1 \mathrm{mg}$ of PDA in $1 \mathrm{~mL}$ DMSO), the resulting gels were a highly heterogeneous morphology, very different from the homogeneous, smooth morphology observed when using DMSO (Figure 
S18). Additionally, the dimensions of COF aerogel were similar to that prepared at lower concentrations due to the mold dimensions, and therefore the density increased significantly. The amount of acetic acid also influenced gelation and aerogel formation. A small amount of $6 \mathrm{M}$ acetic acid (e.g. $0.05 \mathrm{~mL} \mathrm{AcOH}$ in $1 \mathrm{~mL}$ DMSO) added facilitated the synthesis of wet gels while greater amounts (e.g. 0.4 $\mathrm{mL}$ or $0.6 \mathrm{~mL} \mathrm{AcOH}$ in $1 \mathrm{~mL}$ DMSO) resulted in heterogeneous gels (Figure S19). Finally, we found that in the last step drying procedure, freeze drying was not an effective method to recover fragile COFs as it caused partial loss of crystallinity for these COF chemistries studied (Figure S20). This can be attributed to structure distortion caused by water removal during freeze drying process and it is consistent with our previous report that demonstrated that high surface tension solvents can damage the pore structure and reduce crystallinity of COFs. ${ }^{18}$

Absorption performance. Due to the highly porous structures, high surface area, and low density, the COF aerogels are expected to serve as effective absorbents for organic pollutants and show promising applications in oil/water separations. ${ }^{1,6,12}$ To test the ability of the COF aerogels to absorb organic pollutants, we conducted a series of absorption tests for TAPB-OMePDA and TAPB-TFPA COF aerogels. These two COF aerogels were selected as they could be synthesized and crystallized in DMSO solvents directly without further reactivation. In addition, both exhibited very high surface areas (over 1000 $\mathrm{m}^{2} / \mathrm{g}$ ). A piece of TAPB-TFPA COF aerogel was placed at the bottom of a container filled with water and chloroform containing the dye Sudan III. Upon adding the COF aerogel to the container, the chloroform was selectively absorbed within seconds (Figure 3a, Video S1 in Supporting Information). In a similar test, we placed a piece of TAPB-TFPA COF aerogel was placed on the surface of water and silicon oil (dyed with Sudan III), and we observed that the COF aerogel similarly absorbed the silicon oil immediately (Figure $3 b$ ). These simple adsorption processes were effective for completely removing organic and oil phases, leaving only a clean aqueous phase behind.

We measured the absorption capacities of the COF aerogels for various organic solvents (Figure 3c, d). TAPBOMePDA aerogelshowed a very large absorption capacity of approximately 16 - 35 times its own weight, depending on the solvent tested. The largest absorption capacities were measured for ethanol, dichlorobenzene, dioxane, toluene and chloroform (Figure 3c). TAPB-TFPA aerogelexhibited a comparable organic solvents uptake capacity as TAPB-OMePDA aerogel, and it exhibited a better absorption capacity for dichlorobenzene and chloroform than other solvents (Figure 3d). To test the recyclability and re-use of COF aerogels, the aerogels were solvent exchanged using ethanol and dried using $\mathrm{ScCO}_{2}$. The resulting washed and dried aerogels exhibited no loss of absorption capacity (Figure 3e) or loss of crystallinity (Figure 3f) over 10 cycles of absorption, washing, and drying.

Organic dye adsorption. There is a need for low-cost, portable, point-of-use technologies to treat contaminated water, especially in remote or low-resource locations. ${ }^{19}$
Adsorption is an effective and low-cost process that has been widely utilized, but the development of stable and effective adsorbents with hierarchical porosities remains a challenge. ${ }^{20}$ We focused on TAPA-TFPA COF aerogel to test for the removal of micropollutants from water because this COF had a unique morphology with large micropores (see Figure S13a). The TAPA-TFPA COF exhibited excellent performance in the removal of organic pollutant methylene blue (MB), achieving more than $93 \%$ removal efficiency within 13 mins (Figure 4b). By contrast, TAPA-TFPA powders exhibited a much slower removal rate of MB, with a similar removal efficiency only after over one hour (Figure S21). Both the TAPA-TFPA COF aerogel and powders followed a pseudo second order model for adsorption (Figure S22), and the COF aerogel had a pseudo second order rate constant $\left(K_{o b s}\right)$ more roughly four time greater than the powders $\left(0.20 \mathrm{~g} \mathrm{mg}^{-1} \mathrm{~min}^{-1}\right.$ vs $0.048 \mathrm{~g} \mathrm{mg}^{-1} \mathrm{~min}^{-1}$ for the aerogel and powder, respectively). This suggests that the macroporous aerogel structure increases access to micro and macropores in the COF structure. Furthermore, unlike the powder, the aerogel can be easily recovered, washed, and reused for additional adsorption processes. After washing the COF aerogel with acetone and methanol, was utilized for 4 more cycles without any degradation in performance in the removal of MB (Figure 4c). We additionally performed a flow-through experiment to demonstrate the versatility of the COF aerogel in different adsorption treatments. We passed a $10 \mu \mathrm{M}$ MB solution through a column packed with TAPA-TFPA aerogel and measured a 99.0 $\%$ removal efficiency (Figure S23).

We also found that the TAPA-TFPA COF aerogel was effective in the removal of other organic and inorganic contaminants. Specifically, we tested Methyl Blue (MLB), Congro Red (CR), Methyl Orange (MO), and gold nanoparticles (Au NPs) (See Figure S24, Figure S25, Table S3). The removal efficiency for MLB was $97.2 \%$, and the COF had a high $K_{o b s}$ of $0.17 \mathrm{~g} \mathrm{mg}^{-1} \mathrm{~min}^{-1}$. The COF aerogel was also effective for the adsorption of MO and CR, with removal efficiencies of $80.6 \%$ and $87.0 \%$ and $K_{o b s}$ of $0.167 \mathrm{~g} \mathrm{mg}^{-1}$ $\mathrm{min}^{-1}$ and $0.068 \mathrm{~g} \mathrm{mg}^{-1} \mathrm{~min}^{-1}$, respectively. The significantly slower removal rate of $\mathrm{CR}$ can be attributed to its larger molecular size compared with MLB and MB. ${ }^{6}$ Finally, the TAPA-TFPA COF aerogel effectively removed inorganic Au NPs (average size $5 \mathrm{~nm}$ ) from water with a $99.0 \%$ removal efficiency and a high $K_{\text {obs }}$ of $0.60 \mathrm{~g} \mathrm{mg}^{-1} \mathrm{~min}^{-1}$. We hypothesize that the highly efficient removal of Au NPs was due to interactions between the Lewis acid surface of Au NPs and the imine bonds of $\mathrm{COF}^{21,22}$

Iodine uptake. Radioiodine is a pollutant generated by nuclear fission. Iodine is highly volatile and quickly disperses in air, and therefore radioiodine is a serious threat to environmental and personal health. ${ }^{23}$ COFs have been studied for the capture and storage of radiological iodine, ${ }^{23-26}$ and here we investigated the effectiveness of $\mathrm{COF}$ aerogels for the adsorption of radioiodine. We tested TAPB-PDA COFs for the adsorption of iodine, as shown in Figure 5. This COF was chosen because it has large pores $(3.3$ 

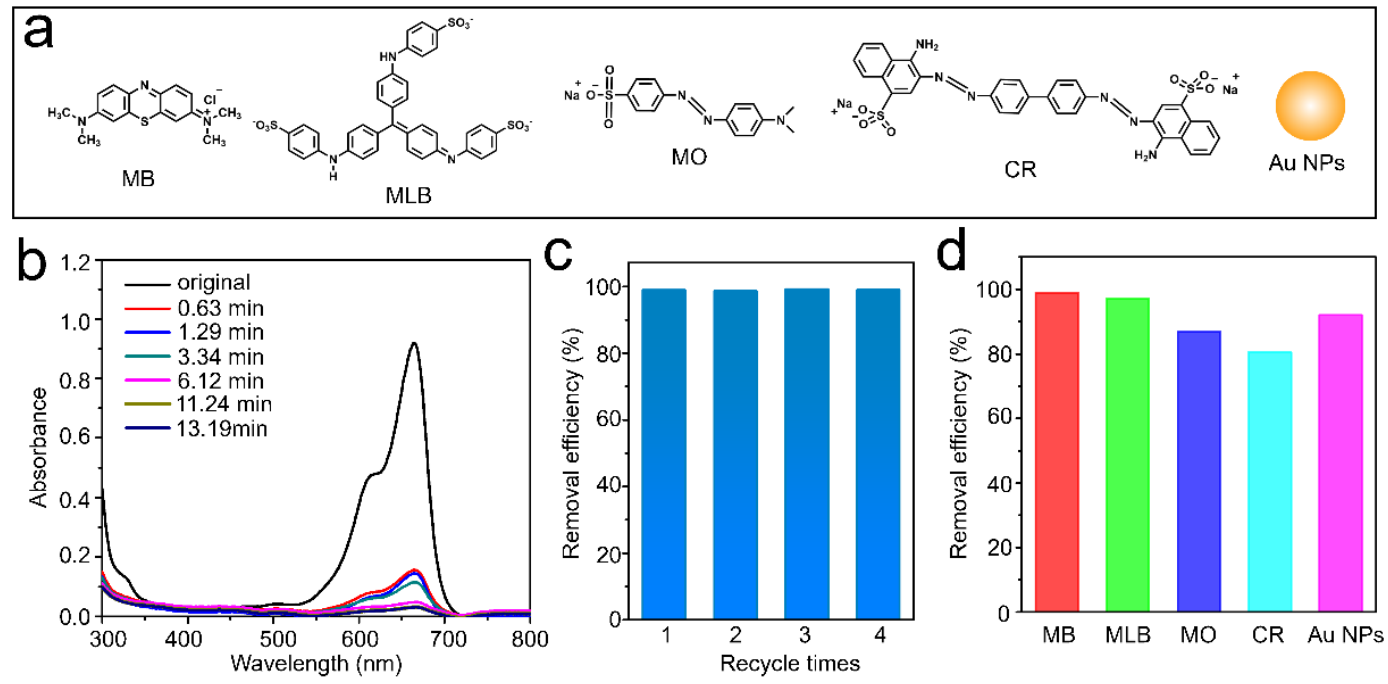

Figure 4. Performance of TAPA-TFPA COF aerogel for the adsorption of organic dyes and gold nanoparticles. (a) Pollutants studied in the adsorption tests. (MB: Methylene blue; MLB: Methyl blue; MO: Methyl orange; CR: Congo red; Au NPs: gold nanoparticles. (b) Time-dependent UV-Vis absorbance measurements during the adsorption of MB (c) Removal efficiency of MB by COF aerogels after multiple adsorption and wash cycles. (d) Removal efficiencies of different dyes by the COF aerogels.
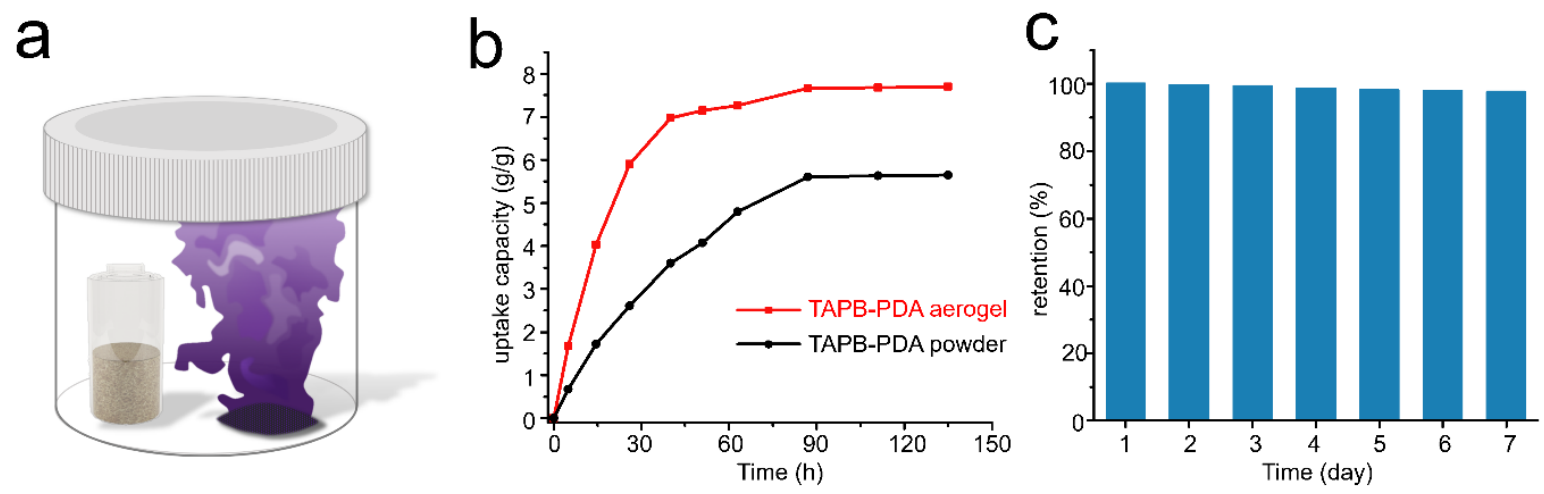

Figure 5. Evaluation of TAPB-PDA COF for iodine adsorption (a) Schematics showing the experimental setup of iodine uptake. (b) Iodine uptake capacity of TAPB-PDA COF aerogel and powder. (c) Measured retention of iodine in the TAPB-PDA COF aerogel after 7 days at ambient conditions.

nm) ${ }^{18,27}$, with no additional substituents or additional pore functionalities that may interfere with the iodine capture process. The TAPB-PDA COF aerogel showed an excellent iodine uptake capacity of $7.7 \mathrm{~g}$ iodine $/ 1.0 \mathrm{~g}$ COF aerogel (Figure 5b). For comparison, we also analyzed the iodine uptake capacity of TAPB-PDA powder. TAPB-PDA powder exhibited lower adsorption kinetics and capacity $(\sim 5.6 \mathrm{~g}$ iodine/1.0 g COF powder, Figure $5 \mathrm{~b}$ ). The better performance of COF aerogel compared with the powders may be due to the lower density and larger total volume of the aerogel, which facilitates the interaction of iodine vapor with the COF intrinsic micropores and macropores. We further measured the ability of the TAPB-PDA COF aerogel to retain iodine. The COF aerogel was removed from the chamber containing iodine and held at ambient temperature and pressure. We detected no loss of iodine over 7 days at ambient conditions (Figure 5c).

\section{CONCLUSIONS}

In summary, we successfully synthesized a series of COF aerogels using DMSO as a solvent and 6M acetic acid as the catalyst. While some COFs were crystalline immediately after synthesis in DMSO and acetic acid, others required an additional reactivation step in dioxane and mesitylene to produce crystalline COF aerogels. These methods produced crystalline, and highly porous COF aerogels with porosities and crystallinities comparable to COF powders produced using conventional solvothermal routes. We characterized the microstructure and morphology of these COF aerogels and demonstrated their potential utility for the absorption of organic solvents, adsorption of organic dyes, gold nanoparticles , and iodine. Due to their lower density and hierarchical pore structure, the COF aerogels showed excellent performance in these tests. This work provides a versatile and widely-applicable strategy to produce COF aerogels and will significantly advance the development of COFs for applications in remediation, separations, and others requiring porous, macroscopic scaffolds. 


\section{ASSOCIATED CONTENT}

Supporting Information. Materials and methods, experimental details, extra characterization data including PXRD, FITR, nitrogen sorption tests and SEM, optimization of COF gel conditions, organic solvent absorption measurements, adsorption tests of dye adsorption and iodine uptake.

\section{AUTHOR INFORMATION}

\section{Corresponding Author}

*(R.V.) E-mail: rafaelv@rice.edu.

\section{Author Contributions}

\#D. Zhu and Y. Zhu contributed equally.

\section{REFERENCES}

(1) Ding, S.-Y.; Wang, W. Covalent Organic Frameworks (COFs): From Design to Applications. Chem. Soc. Rev. 2012, 42 (2), 548568.

(2) Kandambeth, S.; Dey, K.; Banerjee, R. Covalent Organic Frameworks: Chemistry beyond the Structure. J. Am. Chem. Soc. 2019, 141 (5), 1807-1822.

(3) Lohse, M. S.; Bein, T. Covalent Organic Frameworks: Structures, Synthesis, and Applications. Adv. Funct. Mater. 2018, 28 (33), 1705553.

(4) Burke, D. W.; Sun, C.; Castano, I.; Flanders, N. C.; Evans, A. M.; Vitaku, E.; McLeod, D. C.; Lambeth, R. H.; Chen, L. X.; Gianneschi, N. C.; Dichtel, W. R. Acid Exfoliation of Imine-Linked Covalent Organic Frameworks Enables Solution Processing into Crystalline Thin Films. Angew. Chem. 2020, 132 (13), 5203-5209.

(5) Dey, K.; Pal, M.; Rout, K. C.; Kunjattu H, S.; Das, A.; Mukherjee, R.; Kharul, U. K.; Banerjee, R. Selective Molecular Separation by Interfacially Crystallized Covalent Organic Framework Thin Films. J. Am. Chem. Soc. 2017, 139 (37), 13083-13091.

(6) Karak, S.; Dey, K.; Torris, A.; Halder, A.; Bera, S.; Kanheerampockil, F.; Banerjee, R. Inducing Disorder in Order: Hierarchically Porous Covalent Organic Framework Nanostructures for Rapid Removal of Persistent Organic Pollutants. J. Am. Chem. Soc. 2019, 141 (18), 7572-7581.

(7) Dey, K.; H, S. K.; Chahande, A. M.; Banerjee, R. Nanoparticle Size-Fractionation through Self-Standing Porous Covalent Organic Framework Films. Angew. Chem. Int. Ed. 2020, 59 (3), 1161-1165.

(8) Mohammed, A. K.; Usgaonkar, S.; Kanheerampockil, F.; Karak, S.; Halder, A.; Tharkar, M.; Addicoat, M.; Ajithkumar, T. G.; Banerjee, R. Connecting Microscopic Structures, Mesoscale Assemblies, and Macroscopic Architectures in 3D-Printed Hierarchical Porous Covalent Organic Framework Foams. J. Am. Chem. Soc. 2020, 142 (18), 8252-8261.

(9) Dey, K.; Bhunia, S.; Sasmal, H. S.; Reddy, C. M.; Banerjee, R. Self-Assembly-Driven Nanomechanics in Porous Covalent Organic Framework Thin Films. J. Am. Chem. Soc. 2021, 143 (2), 955-963.

(10) Sasmal, H. S.; Halder, A.; Kunjattu H, S.; Dey, K.; Nadol, A.; Ajithkumar, T. G.; Ravindra Bedadur, P.; Banerjee, R. Covalent SelfAssembly in Two Dimensions: Connecting Covalent Organic Framework Nanospheres into Crystalline and Porous Thin Films. J. Am. Chem. Soc. 2019, 141 (51), 20371-20379.

(11) Karak, S.; Kandambeth, S.; Biswal, B. P.; Sasmal, H. S.; Kumar, S.; Pachfule, P.; Banerjee, R. Constructing Ultraporous Covalent Organic Frameworks in Seconds via an Organic Terracotta Process. J. Am. Chem. Soc. 2017, 139 (5), 1856-1862.

(12) Li, C.; Yang, J.; Pachfule, P.; Li, S.; Ye, M.-Y.; Schmidt, J.; Thomas, A. Ultralight Covalent Organic Framework/Graphene Aerogels with Hierarchical Porosity. Nat. Commun. 2020, 11 (1), 4712.

(13) Zhai, L.; Huang, N.; Xu, H.; Chen, Q.; Jiang, D. A Backbone Design Principle for Covalent Organic Frameworks: The Impact of Weakly Interacting Units on $\mathrm{CO} 2$ Adsorption. Chem. Commun. 2017, 53 (30), 4242-4245.
(14) Peng, Y.; Huang, Y.; Zhu, Y.; Chen, B.; Wang, L.; Lai, Z.; Zhang, Z.; Zhao, M.; Tan, C.; Yang, N.; Shao, F.; Han, Y.; Zhang, H. Ultrathin Two-Dimensional Covalent Organic Framework Nanosheets: Preparation and Application in Highly Sensitive and Selective DNA Detection. J. Am. Chem. Soc. 2017, 139 (25), 8698-8704.

(15) Hou, J.; F. Sapnik, A.; D. Bennett, T. Metal-Organic Framework Gels and Monoliths. Chem. Sci. 2020, 11 (2), 310-323.

(16) Makal, A.; Schilf, W.; Kamieński, B.; Szady-Chelmieniecka, A.; Grech, E.; Woźniak, K. Hydrogen Bonding in Schiff Bases - NMR, Structural and Experimental Charge Density Studies. Dalton Trans. 2010, 40 (2), 421-430.

(17) Chaudhari, A. K.; Han, I.; Tan, J.-C. Multifunctional Supramolecular Hybrid Materials Constructed from Hierarchical SelfOrdering of In Situ Generated Metal-Organic Framework (MOF) Nanoparticles. Adv. Mater. 2015, 27 (30), 4438-4446.

(18) Zhu, D.; Verduzco, R. Ultralow Surface Tension Solvents Enable Facile COF Activation with Reduced Pore Collapse. ACS Appl. Mater. Interfaces 2020. 12, 29, 33121-33127.

(19) Garcia-Segura, S.; Qu, X.; J. Alvarez, P. J.; P. Chaplin, B.; Chen, W.; C. Crittenden, J.; Feng, Y.; Gao, G.; He, Z.; Hou, C.-H.; Hu, X.; Jiang, G.; Kim, J.-H.; Li, J.; Li, Q.; Ma, J.; Ma, J.; Brockway Nienhauser, A.; Niu, J.; Pan, B.; Quan, X.; Ronzani, F.; Villagran, D.; David Waite, T.; Shane Walker, W.; Wang, C.; S. Wong, M.; Westerhoff, P. Opportunities for Nanotechnology to Enhance Electrochemical Treatment of Pollutants in Potable Water and Industrial Wastewater a Perspective. Environ. Sci. Nano 2020, 7 (8), 2178-2194.

(20) Luo, J.; Yu, D.; Hristovski, K. D.; Fu, K.; Shen, Y.; Westerhoff, P.; Crittenden, J. C. Critical Review of Advances in Engineering Nanomaterial Adsorbents for Metal Removal and Recovery from Water: Mechanism Identification and Engineering Design. Environ. Sci. Technol. 2021. https://doi.org/10.1021/acs.est.0c07936.

(21) Yazdani, H.; Pardis, S.; Loni, M.; Bazgir, A. Gold Nanoparticle as a Lewis Acid Catalyst in 1,3-Dipolar Cycloaddition Reaction. Catal. Commun. 2020, 134, 105844.

(22) Deng, Q.; Mu, F.; Qiao, Y.; Wei, D. A Theoretical Review for Novel Lewis Base Amine/Imine-Catalyzed Reactions. Org. Biomol. Chem. 2020, 18 (35), 6781-6800.

(23) Wang, P.; Xu, Q.; Li, Z.; Jiang, W.; Jiang, Q.; Jiang, D. Exceptional Iodine Capture in 2D Covalent Organic Frameworks. Adv. Mater. 2018, 30 (29), 1801991.

(24) Li, J.; Zhang, H.; Zhang, L.; Wang, K.; Wang, Z.; Liu, G.; Zhao, Y.; Zeng, Y. Two-Dimensional Covalent-Organic Frameworks for Ultrahigh Iodine Capture. J. Mater. Chem. A 2020, 8 (19), 95239527.

(25) Wang, C.; Wang, Y.; Ge, R.; Song, X.; Xing, X.; Jiang, Q.; Lu, H.; Hao, C.; Guo, X.; Gao, Y.; Jiang, D. A 3D Covalent Organic Framework with Exceptionally High Iodine Capture Capability. Chem. Eur. J. 2018, 24 (3), 585-589.

(26) An, S.; Zhu, X.; He, Y.; Yang, L.; Wang, H.; Jin, S.; Hu, J.; Liu, H. Porosity Modulation in Two-Dimensional Covalent Organic Frameworks Leads to Enhanced Iodine Adsorption Performance. Ind. Eng. Chem. Res. 2019, 58 (24), 10495-10502.

(27) Sick, T.; Rotter, J. M.; Reuter, S.; Kandambeth, S.; Bach, N. N.; Döblinger, M.; Merz, J.; Clark, T.; Marder, T. B.; Bein, T.; Medina, D. D. Switching on and off Interlayer Correlations and Porosity in 2D Covalent Organic Frameworks. J. Am. Chem. Soc. 2019, 141 (32), 12570-12581. 